\title{
Advancing PH Emergency Preparedness Informatics to Support Emergency Responses
}

\author{
Nikolay Lipskiy* and James Tyson \\ Centers for Diseases Control and Prevention (CDC), Office of Public Health Preparedness and Response's (OPHPR), Division of \\ Emergency Operations (DEO), Atlanta, GA, USA
}

\section{Objective}

The purpose of this project is to demonstrate progress in developing a scientific and practical approach for public health $(\mathrm{PH})$ emergency preparedness and response informatics (EPRI) that supports the National Health Security Strategy and Global Health Security Agenda (GHSA) objectives. PH emergency operations centers (EOC) contribute to health security objectives because they operationalize response, recovery and mitigation activities during national and international $\mathrm{PH}$ events. The primary focus of this presentation is to describe the results of an analysis of CDC's EOC, and other EOCs, in building their EPRI capabilities.

\section{Introduction}

Global travel and human migration patterns facilitate the spread of diseases such as influenza $\mathrm{A} / \mathrm{H} 1 \mathrm{~N} 1$, Ebola, and Zika, increasing pressure to $\mathrm{PH}$ systems to protect their constituents against global health threats. Effective prevention, detection, and rapid response to threats rely heavily on adequate information sharing. This requires effective information management through PH EPRI applications such as information systems and tools, knowledge management, and a continuous cycle improvement to maintain system quality. Enhancement of PH EPRI capabilities contributes to improved decision making during emergencies ${ }^{1}$. It transforms public health practice and improves health outcomes through better surveillance, epidemiology, integrated delivery of services, and other emergency preparedness and response activities.

EPRI activities depend on both technical systems and the people who use them. Without adequate training, these systems cannot be effective. CDC's PH EOC information processes and data flows are a notable use case, utilized by hundreds of emergency responders during large-scale PH events. By analyzing this use case, CDC's informaticians have identified multiple opportunities for advancing PH EPRI and advance the objectives of the GHSA.

\section{Methods}

PH EPRI is an interdisciplinary science, incorporating knowledge and techniques from a multiple fields of research and practice. These include epidemiology and surveillance, gathering and distributing information for situational awareness (SA), technology infrastructure development, incident management, and several other disciplines.

CDC's Situational Awareness Branch used three sources for this analysis: direct analysis of CDC's EOC information systems and SA activities; WHO's Framework for a Public Health Emergency Operations Centre ${ }^{2}$, and HHS' Public Health and Medical Situational Awareness Strategy ${ }^{3}$. This assessment also included a comparison of the objectives of PH EPRI to the objectives of other emerging disciplines, such as $\mathrm{PH}$ informatics and emergency preparedness informatics. This helped in avoiding overlap with other disciplines and fixing gaps within PH EPRI.

\section{Results}

The following information flows were identified as part of the CDC's EOC operations: Managing and Commanding, Operations, Planning and Intelligence, Logistics, and Finance/Administration.
These information flows are standard for PH EPRIs. Each information flow is supported by an information structure that consists of hierarchical categories. For example, the Operations information flow includes Task Tracking, Event Investigation, and Controlling. As of August, 2016, CDC's EOC defined 41 hierarchical categories for PH EPRI data flows.

CDC's EOC harmonized different information flows by using a consistent vocabulary to describe the hierarchical components of each information flow. Two hundred thirty six data elements of this vocabulary were harmonized as of August 2016 to standardize its EPRI systems. The hierarchy of PH EOC data flows and harmonized data elements were published in the CDC Vocabulary and Access Distribution System, VADS ${ }^{4}$.

Some information flows were unique to PH EPRI, and were not covered by other emerging disciplines. Examples of these unique information flows include some incident management data, logistics for deployment of $\mathrm{PH}$ personnel and resources, and some event mitigation data.

\section{Conclusions}

CDC's EOC has several harmonized information flows that benefit users and $\mathrm{CDC}$ emergency activations. Understanding these unique PH EPRI data flows helps improve preparedness of staff for working with information flows during emergency activations. Advances in harmonization and standardization helped improve PH EPRI, optimize staff training.

\section{Keywords}

Global health security; PH emergency preparedness and response informatics; Emergency preparedness and response; data harmonization and interoperability

\section{References}

1. CDC. Why is Global Public Health Informatics important to public health? At: http://www.cdc.gov/globalhealth/healthprotection/gphi/ what/objectives.html

2. WHO. Framework for a Public Health Emergency Operations Centre. Interim document. November, 2015. At: http://www.who.int/ihr/ publications/9789241565134 eng/en/

3. Medical Situational Awareness Strategy. HHS, Assistant Secretary for Preparedness and Response (ASPR), 2014. At: http://www.phe.gov/ Preparedness/legal/Documents/phms-sas-20140516.pdf

4. CDC. Public Health Information Network. Vocabulary Access and Distribution System (PHIN VADS). At: https://phinvads.cdc.gov/ vads/SearchVocab.action

\section{*Nikolay Lipskiy}

E-mail: dgz1@cdc.gov 